\title{
EL CONCEPTO DE XOCHIYAOYOTL EN EL MUNDO PREHISPÁNICO SEGÚN LAS RELACIONES DE CHIMALPAHIN CUAUHTLEHUANITZIN
}

\author{
Laura Alicino ${ }^{\mathrm{a}, \mathrm{b}}$ \\ aKing's College London, Modern Language Centre, 170 Strand, London WC2R 2LS, United Kingdom \\ bDipartimento di Lingue, Letterature e Culture Moderne (LILEC), Università di Bologna, via Cartoleria n. 5, Bologna 40124, Italy
}

\begin{abstract}
La guerra florida es considerada un conflicto en el cual se enfrentaron, durante muchos años, la Triple Alianza Azteca y los reinos del valle tlaxcalteco-poblano. Por mucho tiempo la xochiyaoyotl se ha estudiado como una guerra ritual, prolongada en el tiempo y basada en un recíproco acuerdo. Según las teorías críticas que avaloran esta tesis, el fin de la xochiyaoyotl era obtener las víctimas cuyo sacrificio proveía la alimentación de los dioses. Sin embargo, en las últimas décadas del siglo XX, gracias al descubrimiento y a la traducción de nuevos documentos relativos a la historia prehispánica, el concepto ritual y religioso de la xochiyaoyotl ha empezado a cuestionarse. La fuente que otorga más detalles sobre la xochiyaoyotl es la obra del cronista chalca Domingo Chimalpahin. Este testimonio da evidencia de enfrentamientos llamados xochiyaoyotl precedentes al reinado de Motecuhzoma Ilhuicamina, en que estuvieron involucrados primeramente chalcas y tlacochcalcas y, después, los mexicas. A través del análisis del testimonio de Chimalpahin este estudio busca matizar las teorías acerca de la finalidad puramente ritual de la xochiyaoyotl, avanzando como hipótesis que los mexicas se adueñaron del concepto de xochiyaoyotl, desarrollándolo conforme a las nuevas necesidades políticas sobrevenidas.
\end{abstract}

Según Bueno Bravo (2009), hasta nuestros días la mayoría de los trabajos dedicados al estudio de la América prehispánica se han concentrado, por la mayor parte, en los aspectos religiosos y mitológicos. Otros rasgos como la economía, la estructura del poder y la guerra, se han investigado menos. Sin embargo, las guerras floridas (xochiyaoyotl) representan una excepción. Desde el momento de la llegada de los españoles a México en 1519, el concepto de xochiyaoyotl ha fascinado toda clase de curiosos de las antigüedades indígenas y se han publicado muchos trabajos al respecto.

La mayoría de las fuentes (Durán 1967, 1980; Códice Ramírez, Alvarado Tezozómoc 1987) y sus respectivas interpretaciones (Caso 1961; Soustelle 1965; Davies 1966), presentan la guerra florida como un conflicto pactado en el que se enfrentaron durante muchos años los reinos del valle tlaxcalteco-poblano (entre ellos Tlaxcala, Huexotzinco y Cholula) y la Triple Alianza azteca. Esta alianza fue la última confederación de estados antes de la llegada de los españoles, y estaba formada por las ciudades-estado de Tenochtitlan y Tetzcoco, de filiación nahua, y Tlacopan, de cultura otomí.

Según el reciente estudio de Velasco Murillo (2016), también en Zacatecas existen evidencias de estas tipologías de enfrentamientos rituales pactados llamados saçemis que se pueden definir como "large-scale fights (often lethal) between opposing indigenous groups that occurred outside civic sponsorship or religious ceremonial cycles and thus had no political consequences" (Velasco Murillo 2016:48). Asimismo, el estudio de Velasco proporciona

laura.alicino@kcl.ac.uk detalles interesantes acerca de enfrentamientos muy similares a la guerra florida y a los saçemis en la región de los Andes. De hecho, en las crónicas coloniales de Bernabé Cobo, José de Acosta, Juan de Betanzos, Inca Garcilaso de la Vega y Pedro Gutiérrez de Santa Clara, se mencionan enfrentamientos rituales llamados tinkus durante la época incaica (Velasco Murillo 2016:51).

Conforme a las fuentes y teorías críticas que avaloran la tesis ritual, el fin de la xochiyaoyotl era obtener cierto número de víctimas, cuyo sacrificio proveía el alimento de los dioses: la sangre humana. Sin embargo, a partir de las últimas décadas del siglo XX, gracias al descubrimiento y a la traducción de nuevos documentos relativos a la historia prehispánica, el concepto ritual y puramente religioso de la xochiyaoyotl ha empezado a ser cuestionado, así como la fecha precisa de su invento. La fuente que nos otorga más detalles inéditos y extremamente interesantes sobre la guerra florida es, sin duda, la obra del cronista chalca Domingo Francisco de San Antón Muñón Chimalpahin Quauhtlehuanitzin.

Chimalpahin nació en 1579 descendiente de la nobleza chichimeca del reino de Tzacuatitlan-Amaquemecan-Chalco. Hacia el final del siglo XVII redacta en lengua náhuatl la historia de Chalco, su altepetl de origen, eligiendo la forma del xiuhpohualli, o anales. El género es dominante entre los pueblos nahuas antes de la llegada de los españoles. Aparece durante el reinado de Itzcoatl (1427-1440), cuando se consolida la dominación mexica. Impuesto en todos los territorios conquistados, según declara Benoist (2003), al principio el xiuhpohualli se identifica como un elemento esencial de la conquista azteca. Paradójicamente, más tarde se convertiría en un instrumento de salvaguarda de la identidad loca; una forma de resistencia al imperialismo mexica. Cada altepetl 
posee su propio xiuhpohualli en el que se narra la genealogía de la élite de la comunidad, junto a los acontecimientos históricos fundamentales, como migraciones, fenómenos atmosféricos y guerras.

La obra de Chimalpahin se divide en ocho Relaciones a las que se añaden el Diario y el Memorial de Culhuacan, partes integrales del mismo. Las obras se basan en varias tipologías de fuentes. Romero Galván (1977) identifica por lo menos dos grupos: (a) fuentes indígenas que comprenden documentos escritos en lengua náhuatl con caracteres latinos, códices pictográficos y testimonios orales; y (b) libros impresos sobre geografía e historia española y universal (ver también Messiaen 2003).

El presente ensayo intenta estudiar la evolución del concepto de xochiyaoyotl, desde los testimonios religioso-rituales hasta las revisiones más recientes, proporcionando también nuevos detalles acerca de este fascinante tema. Demostraré que, en el período del fulgor del imperio azteca, los mexicas se adueñaron del concepto de xochiyaoyotl desarrollándolo y reinterpretándolo conforme a las nuevas necesidades políticas. En primer lugar, analizaré las fuentes en que se han basado las sucesivas interpretaciones rituales de las guerras floridas, desde el reino de Motecuchzoma Ilhuicamina hasta el reino de Motecuhzoma Xocoyotzin. Luego, procederé al análisis de los testimonios relativos a la xochiyaoyotl precedentes al auge del dominio azteca, así como en las Relaciones de Chimalpahin y otras fuentes.

\section{MESOMAERICA Y LA CONCEPCION SAGRADA DE LA GUERRA}

La guerra ( yaoyotl) constituía un elemento fundamental en la vida de cada pueblo mesoamericano, con implicaciones tanto de carácter religioso, como de carácter socio-político. Taube (1992) ha evidenciado la presencia del concepto de guerra sagrada en Teotihuacan ya durante el Clásico temprano. Siguiendo las interpretaciones de Caso y Bernal (1952), Taube sostiene que una de las cabezas de piedra que se repiten en la fachada del Templo Viejo de Quetzalcoatl en Teotihuacan encarna una serpiente. Más precisamente, se trata de una representación primigenia del Postclásico Xiuhcoatl, o sea la culebra de fuego y turquesa que en la cultura azteca era el arma de Huitzilopochtli. Taube añade que en la iconografía teotihuacana esta serpiente representaba tanto la guerra como el fuego (Taube 1992:59). La misma simbología se encuentra también entre los mayas y los zapotecas del Clásico temprano y tardío, como muestra tanto la arquitectura de Tikal como la de Oaxaca. Es interesante notar que, según Taube, la Serpiente de la Guerra en Tikal se consideraba un elemento foráneo y perteneciente precisamente a la tradición teotihuacana:

\begin{abstract}
Many researchers have noted that much of the Teotihuacan style iconography found in the Maya region is based on war. [...] on the "warrior stelae" of Piedras Negras, local Maya rulers consciously identified themselves with a war complex from Teotihuacan. [...] it is clear that the Teotihuacan imagery represents not foreign invasion, but a local adoption and manipulation of Teotihuacan war regalia and iconography (Taube 1992:74).
\end{abstract}

En la sucesiva ideología nahua, la noción de la guerra sagrada procedía directamente de la mitología. Según narran las fuentes el Quinto Sol, nacido en una pira encendida exactamente en Teotihuacan, es el sol originado gracias al auto-sacrificio de los dioses. En la Leyenda de los soles (Velázquez 1975a) se lee que después de que Nanahuatzin, el dios buboso, se trasforma en astro solar, los demás dioses se dan cuenta de que él no se movía: “Dijeron los dioses: «¿Por qué no se mueve?» [...] Respondió el Sol: «Porque pido su sangre y su reino»" (Velázquez 1975a:121). Como el sol pedía un sacrificio de sangre para que este le permitiera moverse, todas las divinidades se sacrifican para proveer este nutrimiento.

Siempre en la Leyenda de los soles se describe como la guerra y el sacrificio representan el fundamento de la vida. De hecho, después de la creación de los mixcohua:

“[...] llamó el Sol a los cuatrocientos mixcohua, les entregó flechas y les dijo: "He aquí con que me serviréis de comer y me daréis de beber. [...] pero no hicieron su deber [...] se divirtieron $[\ldots]$ A veces cojen un tigre y no lo dan al Sol [...] durmieron con mujeres $[\ldots]$ y anduvieron enteramente beodos" (Velázquez 1975a:123).

Por esta razón, el Sol llama a los cinco mixcohua nacidos a la postre y les ordena matar a los que no habían cumplido con su tarea: "y fué la oportunidad de que se hicieran guerra" (Velázquez 1975a:123). En la Historia de los mexicanos por sus pinturas (Garibay 1965:90), también se exalta el valor de la guerra y de los sacrificios provenientes de ella como sustento de la vida:

\begin{abstract}
"Un año después que el sol fue fecho, que fue primero del tercero treze después del diluvio, Camasale, uno de los cuatro dioses, fue al octavo cielo, y crió cuatro hombres y una muger por hija, para que diesen guerra y oviese corazones para el sol y sangre que bebiese [...]."
\end{abstract}

Como podemos comprobar, la guerra constituía un deber cósmico. Soustelle (1965) asevera que como los dioses pagan con su sangre el nacimiento del mundo, los hombres tienen que repagar su deuda de sangre para que el sol permanezca en movimiento y la vida pueda continuar. Haciendo la guerra, pues, los hombres se conformarían a la voluntad de los dioses desde el origen del mundo.

En los códices pictográficos pertenecientes a la cultura mexica, la guerra se representaba por medio del glifo atl-tlachinolli. El difrasismo se lee literalmente "agua quemada" o "agua preciosa", o sea la sangre. Se trata de una metáfora cuyo glifo se repite constante y obsesivamente, por ejemplo, en los relieves del Teocalli de la Guerra Sagrada, así como comenta Caso (1927). El Teocalli de la Guerra Sagrada es un monolito encontrado a fines de julio de 1926 en el Palacio Nacional de la Ciudad de México y actualmente se encuentra en el Museo Nacional de Antropología. El estudio de Caso destaca un elemento fundamental: los glifos del atl-tlachinolli se encuentran frente a la boca de cada divinidad impresa en el monolito. Se trata de una posición que en los códices indígenas identifica la palabra o el canto (Caso 1927:31), indicándonos que son precisamente los dioses quienes están pidiendo la guerra sagrada. Caso, de hecho, supone que la piedra sea otro monolito más dedicado al culto del Sol y de la guerra. Sin embargo:

[...] estas dos ideas, Sol y guerra sagrada, se acompañan neces-
ariamente en el pensamiento azteca; pues el Sol necesita para
moverse el sacrificio que le proporciona su alimento de sangre
y corazones humanos, y el sacrificio requiere, a su vez, la
guerra (Caso 1927:62).

Sin embargo, aunque justificada por la cosmogonía, la guerra tenía también claros significados políticos y económicos. Zantwijk (1962) asevera que, entre los mexicas, la guerra 
simbolizaba el medio de conquista para sujetar todo el mundo a un orden cósmico de que ellos se suponían portadores. La conquista se consideraba un derecho heredado. De hecho, toda tipología de guerra estaba regulada por una jurisdicción precisa e inmutable que se manifestaba también en el ordenamiento social del pueblo mexica. El guerrero se posicionaba casi en la cúspide de la pirámide social y es interesante considerar que, según algunas fuentes, las órdenes de los guerreros águilas y jaguares aztecas se engendraron precisamente en la pira de Teotihuacán (Velázquez 1975a; Sahagún 1989).

\section{EL ORIGEN DEL CONCEPTO RITUAL Y RELIGIOSO DE LA XOCHIYAOYOTL}

Los testimonios acerca de la invención ritual y religiosa de la xochiyaoyotl y de la motivación por la que se concibió son varios, pero muchas veces discordantes. Esa primera interpretación que considera la guerra florida como una práctica ritual se relaciona, indiscutiblemente, con la evidencia histórica de los continuos enfrentamientos militares entre la Triple Alianza Azteca y los pueblos del Valle tlaxcalteco-poblano.

Al fin de proporcionar conclusiones exhaustivas, en esta sección analizo cada una de las fuentes. Es interesante notar que las versiones cambian con respecto a la procedencia geográfica de los cronistas, o sea al ser mexicas, texcocanos o tlaxcaltecas. Este es un punto central a considerar para un análisis puntual del concepto de guerra florida.

Según declara Durán (1967) en la Historia de las Indias de la Nueva España, la invención de la xochiyaoyotl se da durante el reino de Motecuhzoma Ilhuicamina (1440-1468), bajo propuesta de su consejero Tlacaelel. Después de la construcción del nuevo templo dedicado al dios Huitzilopochtli, Tlacaelel promueve la institución de una "feria militar" (Durán 1967:240) contra los reinos de Tlaxcala, Cholula y Huexotzinco. La idea procede de la necesidad de obtener frecuentes víctimas para los sacrificios humanos. Como la mayoría de las provincias ya estaban sujetadas a Mexico-Tenochtitlan, no existía una concreta posibilidad de rebelión para encaminar una guerra de conquista. Según Durán, se establece una nueva ley: cada señorío sujeto a MexicoTenochtitlan y, por consiguiente, a las demás cabeceras de la Triple Alianza, estaba obligado a enviar sus guerreros. Ellos tienen que distinguirse en guerra y asegurar el mayor número de cautivos para el sacrificio, en obediencia al principio según el cual:

"[...] la gloria y honra y grandeza mandamos que no se herede por vía de nacimiento de grandes, sino por vía de guerra y valentía que en la guerra de Tlaxcala, Huexotzinco, Cholula, Atlixco, Tecoac, Tlilqiuhtepec de hoy más se hiciere y ejercitare" (Durán 1967:240).

Como refiere Diego Durán, en el momento mismo en que Motecuhzoma Ilihuicamina acepta la propuesta de Tlacaelel, se informan de la nueva ley a los tetecutin de todos los pueblos sujetos a los mexicas, además de los señores de Huexotzinco y Tlacopan. Claramente, lo mismo se lee en el Códice Ramírez (Alvarado Tezozómoc 1987:132), que representa el núcleo de la historia de Durán:

Traidos y entregados los cautivos de Huaxacac para sacrificar en el dia de la dedicacion del templo, viendo Motecuzuma y Tlacaellel que ya eran tenidos y temidos por toda la tierra y por esto cesarian las guerras, y que cesando ellas cesaria el sacrificar hombres $[\ldots]$ para que esto no faltase $[\ldots]$ fué por orden de Tlacaellel $[\ldots]$ y fué el parecer que pues los Tlaxcaltecas y todas aquella provincia estaban mal con ellos, que fuessen los soldados Mexicanos á los tianguez todos los dias que los hubiera en la provincia de Tlaxcallan, como era en Tlaxcallan, Huexotzinco, Cholula, Atlixco, Tliliuhquitepec y Tecoal, y que de allí en lugar de comprar joyas, comprassen con su sangre víctimas para sus Dioses, lo cual comunicado con el Rey, le pareció muy bien a él y á su consejo, porque demas de tener víctimas para sacrificar á su Dios, seguiase otro bien á la provincia mexicana, que era estar de continuo ejercitados en las armas y en las cosas de la guerra, que para conservación de sus Reynos era lo que más convenía.

Una versión semejante de los hechos se encuentra narrada también en la Crónica Mexicana de Alvarado Tezozómoc (1975:184):

\begin{abstract}
Dixo Tlacaeleltzin a Montecuma: «Senor, pareçe que os aflixís y fatigáis por el sacrificio de estos hijos del sol benidos de Guaxaca y mixtecas y los demás <que $>$ son. Yo personal mente ando con el ojo largo a la priesa de los albañís, canteros que andan $<$ en $>$ la obra y acabami <ento $>$ del gran cu y su brasero y asentaderos de los demás dioses tenedores y sustentadores del çielo. Acabado $<$ que $>$ sea, con gran solenidad, fiesta, rregozijo de todo Mexico Tenuchtitlan y prençipales que a ello serán llamados, se hará y cumpliré buestro deseo y boluntad, que a de ser comprado el gran brasero con $\mathrm{n}<$ uest $>$ ro puro trauajo, sangre, cansançio, y a de ser un gran chalchiliuitl, ancho, grueso y la plumería de ofrenda muy ancha y Iarga, de más de una braça, benida del cabo del mundo, pues perteneçe a $\mathrm{n}<$ uest $>\mathrm{ra}$ abusión (tetzahuitl) Huitzilopochtli; que luego con esto llamaremos a los que están tras de estos montes y montañas, los de Huexocingo y Atxisco, Cholula y Tlaxcala, Tliliuhquitepec y tecoaca y los deyupicotlaca, <que $>$ son muy lexos, y los atraeremos a $\mathrm{n}<$ uest $>$ ra boluntad aunque los acarreemos como con rrecuas de $\mathrm{n}<$ uest $>$ ros puros pies $\mathrm{y}$, sobre el caso, guerra cruel con ellos y tener basallaxe de ellos y tener que sacrificar a $\mathrm{n}<$ uest $>$ ros dioses, porque para yr a Cuextlan es muy lexos y más lo es en Mechuacan.
\end{abstract}

Según subraya Isaac (1983), es interesante notar también que Alvarado Tezozómoc refiere un diálogo entre Motecuhzoma y Tlacaelel en que emerge la existencia previa de un intento imperialista del reino mexica con respecto a las tierras del valle tlaxcalteco-poblano.

En relación a estas primeras fuentes analizadas, cabe destacar que la decisión de prolongar estas guerras cíclicas parece deberse únicamente a los reinos de la "Triple Alianza." No se hace mención alguna de una eventual participación de Tlaxcala, ni de los demás reinos del valle tlaxcalteco-poblano.

Disonantes resultan las declaraciones del cronista texcocano Fernando de Alva Ixtlilxochitl (Isaac 1983). A pesar de que atribuya, como lo hacen los cronistas mexicas, la invención de la xochiyaoyolt a Tlacaelel, considera que esta invención no se dio a causa de la terminación del nuevo templo dedicado a Huitzilopochtli, sino a causa de la famosa y terrible carestía del año 1 tochtli (1454), que duró cuatro años y afectó a toda la tierra fría del altiplano central, al valle de México y algunos territorios del Valle tlaxcalteco-poblano. En esta versión de Ixtlilxochitl (1985) se indica que los señores de Mexico-Tenochtitlan, Texcoco, Tlacopan y del reino de Tlaxcala se juntan para encontrar una solución común al problema de la carestía:

"Y aunque Nezahualcoyotzin en su tierra y reino, Motecuhzomatzin y Totoquihuatzin en los suyos, hicieron todo 
lo posible por socorrer a sus súbditos y vasallos [...], viendo que no cesaba la calamidad se juntaron todos tres con la señoría de Tlaxcalan a tratar el remedio más conveniente para este efecto: los sacerdotes y sátrapas de los templos de México dijeron, que los dioses estaban indignados contra el imperio y que para aplacarlos convenía sacrificar muchos hombres y que esto se había de hacer ordinariamente, para que los tuviesen siempre propicios. Nezahualcoyotzin que era muy contrario a esta opinión, después de haber hecho muchas contradicciones, dijo que bastaba que les sacrificasen los cautivos en guerra, que así como así habían de morir en batalla, se perdía poco, además de que sería muy grande hazaña de los soldados haber vivos a sus enemigos, con lo cual, a más de que serían premiados, harían este sacrificio a los dioses: replicaron los sacerdotes, que las guerras que se hacían eran muy remotas y no ordinarias, que vendrían muy a espacio y debilitados los cautivos que se habían de sacrificar a los dioses, habiendo de ser muy de ordinario y la gente reciente y dispuesta para el sacrificio de los dioses, como lo solían hacer con sus hijos y esclavos" (Ixtlilxochitl 1985:XLI).

Nezahualcoyotl, tlatoani de Texcoco, propone elegir el campo entre Quauhtepec y Ocelotepec, para llevar a cabo esa guerra ritual contra Tlaxcala, Huexotzinco y Cholula. Según narra Ixtlilxochitl, en Texcoco deciden combatir cada mes respectivamente contra Tlaxcala, Huexotzinco y Cholula, para luego recomenzar:

\begin{abstract}
"Así se comenzaron estas guerras y abominables sacrificios de los dioses (o para mejor decir) demonios, hasta que vino el invictísimo don Fernando Cortés primer Marqués del Valle a plantar la santa fe católica [...]. En el año se hacían dieciocho fiestas principales a los dioses fingidos, que era a los primeros días de sus dieciocho meses con que repartían sus años solar en los cuales sacrificaban los nombres cautivos en las guerras referidas y otras fiestas que tenían movibles" (Ixtlilxochitl 1985:XLI).
\end{abstract}

Los mismos detalles proporcionados por Ixtlilxochitl se encuentran también narrados en la Relación de Tetzcoco de Pomar (1975), otro cronista texcocano.

Junto a estos testimonios que se refieren al reinado de Motecuhzoma Ilhuicamina (quinto rey de Mexico-Tenochtitlan), tenemos que considerar las fuentes que relatan el principal acontecimiento que llevó a la interpretación ritual de la xochiyaoyotl. A la hora de llegar a Mexico-Tenochtitlan, Andrés de Tapia, capitán del ejército de Hernán Cortés, pregunta a Motecuhzoma Xocoyotzin (séptimo rey de Mexico-Tenochtitlan) la razón por la que nunca lograron los mexicas conquistar la provincia de Tlaxcala. Motecuhzoma responde:

"Bien lo pudiéramos hacer; pero luego no quedara donde los mancebos ejercitaran sus personas, sino lejos de aquí: y también queríamos que siempre oviese gente para sacrificar a nuestros dioses" (Tapia 1858:245).

La misma conversación y las mismas motivaciones se refieren en la mayoría de las fuentes mexicas y texcocanas ya mencionadas. Reportamos, por ejemplo, dos pasajes de las obras de Diego Durán:

"Y porque viene aquí a coyuntura, quiero decir a qué fin se ordenaban las guerras que entre México y Tlaxcallan y toda la generación tlaxcalteca había. Porque, como muchas veces habremos oído, con mucha facilidad sujetaban los mexicanos a Tlaxcallan y a Huexotzinco, y a Tepeaca y a Tecalla y a Calpa, Cuauhtinchan, Acatzinco, Cuauhquecholan y Atlixco, como habían sujetado a todo lo restante de la tierra. Pero no querían, por dos razones que daban los reyes de México: la primera y principal era decir que querían aquella gente para comida sabrosa y caliente de los dioses, cuya carne les era dulcísima y delicada, y la segunda era para ejercitar sus valerosos hombres, y donde fuese conocido el valor de cada uno. Y así, en realidad de verdad, no se hacían para otro oficio ni fin las guerras entre México y Tlaxcallan, sino para traer gente de una parte y de otra, para sacrificar" (Durán 1980:132).

"La causa porque se movían así tantos a la guerra, aunque la principal era su propio interés y ganancia de honra y bienes; lo segundo era no tener su vida en nada y tener por bienaventurados a los que en la guerra morían y así llamaban a la guerra xochiyaoyotl, que quiere decir 'guerra florida', y por el consiguiente, llamaban a la muerte del que moría en guerra xuchimiquiztli, que quiere decir "muerte rosada, dichosa y bienaventurada" (Durán 1967:205).

Sin embargo, a la hora de confrontar estos testimonios con el punto de vista tlaxcalteco, emergen diferencias que no se pueden ignorar. En primer lugar, Bernal Díaz del Castillo registra en su crónica un diálogo entre Hernán Cortés y dos nobles de Tlaxcala: Xicotencatl y Maxixcatzin. Cortés pregunta por qué los mexicas nunca lograron conquistar la provincia tlaxcalteca, a pesar del gran número de guerreros a su disposición, según antes le habían referido los dos hombres. La respuesta es interesante:

"Y Cortés les dijo: Pues con tanto guerrero que decís que venían sobre vosotros, ¿cómo nunca os acabaron de vencer? Respondieron que aunque algunas veces les desbarataban y les mataban y llevaban muchos de sus vasallos para sacrificar, que también de los contrarios quedaban en el campo muchos muertos y otros presos; y que no venían tan encubiertos que de ello no tuviesen noticia; y cuando lo sabían se apercibían con todos sus poderes, y con la ayuda de los de Huexocingo se defendían y ofendían. Que como todas las provincias y pueblos que ha robado Montezuma y puesto debajo de su dominio están muy mal con los mejicanos, y traían de ellos, por fuerza a la guerra, no peleaban de buena voluntad, antes de los mismos tenían avisos, y que a estas causas les defendían sus tierras lo mejor que podían [...]" (Díaz del Castillo 1968:107).

En segundo lugar, la principal fuente tlaxcalteca, o sea la obra del cronista Muñoz Camargo (1892), provee una explicación diferente acerca de las motivaciones con que los mexicas llevaron la guerra a la provincia de Tlaxcala. El cronista señala que, aunque había continuas guerras entre mexicas y tlaxcaltecas, a pesar de las motivaciones que Motecuhzoma ofrecía a los españoles (motivaciones que él parece conocer), ambas partes sufrían muchas pérdidas. Además, Muñoz Camargo representa a los tlaxcaltecas más bien como víctimas de las ambiciones imperialistas del reino mexica:

"Viendo los de Tlaxcala que de todos puntos se declaraba la enemistad con ellos de parte de los Tenuchcas, procuraron defender su partido como pudieron [...] Puestos en esta controversia enviaron a los Príncipes Mexicanos embajadores, diciéndole que cual había sido la causa que contra ellos se tratase guerra, no habiendo dado ocasión para ello [...] Fueles respondido por los Tenuchcas «Que el Gran Señor de Mexico era Señor Universal de todo el Mundo, que todos los nacidos eran sus vasallos, que a todos los había de reducir para que le reconociesen por Señor [...] A lo cual respondieron los embajadores de Tlaxcala: «Señores muy poderosos, Tlaxcala no os debe vasallaje, ni desde que salieron de las siete cuevas, jamás reconocieron 
con tributo ni pecho a ningún Rey ni Príncipe del Mundo, porque siempre los Tlaxcaltecas han conservado su libertad [...]" (Muñoz Camargo 1892:XIII).

Desde la larga comparación de estas fuentes, emergen evidencias que nos llevan a revisar los estudios (Caso 1961; Davies 1966; Soustelle 1965) que interpretan a la xochiyaoyotl sólo como una guerra ritual, perpetuada por recíproco acuerdo, a fin de aprovisionar cautivos necesarios al sacrificio. En primer lugar, a pesar del testimonio texcocano, ni las fuentes mexicas, ni la tlaxcalteca de Muñoz Camargo, mencionan un pacto previo entre las partes destinadas a enfrentarse en la "guerra florida." Además, el testimonio tlaxcalteca parece sostener la presencia de un proyecto imperialista azteca, lo cual sobresale también en algunos pasajes de las fuentes mexicas de Durán y Alvarado Tezozómoc (para mayores informaciones sobre los enfrentamientos rituales entre mexica y tlaxcaltecas, ver: Isaac 1983).

En segundo lugar, sólo las fuentes texcocanas apoyan la teoría del invento de la xochiyaoyotl como respuesta a la gran carestía de 1454, teoría en seguida aceptada por Harner (1977) y Soustelle (1965). Con respecto a este punto, Hassig (1981) ha demostrado que una de las mayores consecuencias sociales de la carestía del año 1 Tochtli no fue la expansión territorial, sino más bien la intensificación de la actividad agrícola a través de la técnica de la chinampa.

También en las fuentes mexicas se observan versiones discordantes. El Códice Ramírez, y por consiguiente Durán, aceptan que la invención de la xochiyaoyotl se dio por parte de Tlacaelel y subrayan dos finalidades de su ejercicio: (a) proveer cautivos diarios para los sacrificios en ocasión de la finalización del nuevo templo dedicado a Huitzilopochtli; y (b) servir de arena para el entrenamiento constante en el arte de la guerra de los hijos de los pipiltin, o sea los integrantes de la nobleza mexica.

Durán, por su parte, subraya que el invento de estas guerras se da porque no se podían esperar revueltas, a partir de las cuales se podían emprender guerras de conquista, siendo el territorio del Valle de México por la mayoría bajo el poder mexica. Con respecto a ese punto, un estudio reciente de González (2011) acerca del culto de Xipe Tótec en la cultura nahua nos proporciona algunas informaciones interesantes. El culto de Xipe Tótec, "Nuestro señor el desollado", se asociaba a una de las fiestas más significativas, la del tlacaxipehualitzi. En ella se celebraban las victorias militares y se conmemoraba el nacimiento del Quinto sol. Durante dicha fiesta se sacrificaban muchas víctimas, sobre todo guerreros enemigos, a través de la práctica del desollamiento. González subraya que las fuentes, en su mayoría, apuntan a que fue precisamente durante el reino de Motecuhzoma Ilhuicamina cuando más se desarrollaron las fiestas dedicadas a Xipe Tótec. Esto indica que estaban relacionadas a un gran número de campañas militares de conquista (Hicks 1979).

A la luz de esta evidencia, las declaraciones de Tlacaelel acerca de la institución de la xochiyaoyotl porque no se podía esperar ninguna revuelta necesita ser cuestionada o, por lo menos, matizada. Asimismo, observamos que en ninguna de estas dos fuentes aparece el término xochiyaoyotl, que sólo emerge algunas veces en el testimonio de Alvarado Tezozómoc (Hicks 1979). Por su parte, Alvarado Tezozómoc subraya un intento imperialista previo a la terminación del nuevo templo de la principal divinidad azteca, Huitzilopochtli.

En tercer lugar, las fuentes mexicas informan acerca de la necesidad de disponer hasta de nuevas leyes con respecto a la decisión de emprender una feria militar contra Tlaxcala y los demás reinos del Valle tlaxcalteco-poblano (Durán 1980). Dichas leyes, compartidas por la Triple Alianza, prometían riqueza y gloria a todos los guerreros que lograban traer cautivos, so pena de muerte para quien osara rebelarse al dictamen. Podemos concluir, entonces, que la necesidad de establecer una ley específica procedía de la conciencia de que no todos los pueblos sometidos a los aztecas eran partidarios de su política imperialista, según nos refiere el testimonio de Díaz del Castillo (1968).

\section{DESCRIPCION DE LA XOCHIYAOYOTL EN LAS RELACIONES DE CHIMALPAHIN}

La contribución del testimonio de Chimalpahin es fundamental para abrir nuevas perspectivas acerca de la interpretación de la xochiyaoyotl. Durante mucho tiempo, los manuscritos del autor chalca han permanecido divididos y traducidos parcialmente a lenguas diferentes, lo cual no ha permitido una visión completa de su obra. Las traducciones de la obra de Chimalpahin que se consultaron para el presente estudio son las siguientes: traducciones al francés: (a) Annales de Chimalpahin: sixième et septième relation (1258-1612), edición y traducción de Rémi Siméon (1889); y (b) Trosième relation de Chimapahin Quauhtlehuanitzin, edición y traducción de Jacqueline de Durand-Forest (1987). Traducciones al español: (a) Primer Amoxtli Libro. $3^{a}$ Relación de las différentes histoires originales, edición y traducción de Víctor del Castillo (1991); (b) Las ocho relaciones y el memorial de Culhuacán, edición y traducción de Rafael Tena (1998); y (c) Séptima Relación de las Différentes Histoires Originales, edición y traducción de Josefina García Quintana (2003). Para mayores informaciones acerca de las varias traducciones de las obras de Chimalpahin confrontar José Rubén Romero Galván (1976).

Chimalpahin es el primero en mencionar directamente algunos enfrentamientos llamados xochiyaoyotl en un período precedente al reino de Motecuhzoma Ilhuicamina (Tabla 1). En segundo lugar, el cronista chalca nos proporciona una diferencia substancial entre la guerra florida y la guerra propiamente de conquista, a la que llama cocoltic yaoyotl, denominada como guerre meurtière, o guerra homicida, según la traducción de Rémi Siméon (1889).

En esta segunda parte del ensayo, intento analizar todos los pasajes de las ocho Relaciones de Chimalpahin en que aparece mencionada la xochiyaoyotl, al fin de facilitar un cuadro cronológico sobre el cual propondré un nuevo punto de vista acerca de la invención de la guerra florida, y de su interpretación entre los pueblos mexicas prehispánicos.

En su crónica, Chimalpahin relata la historia de Chalco, desde su fundación hasta su sometimiento a Mexico-Tenochtitlan. Con este

Tabla 1. Cuadro cronológico de la Xochiyaoyotl según las Relaciones de Chimalpahin

\begin{tabular}{lll}
\hline \hline Año & Mención & Pueblos involucrados \\
\hline 1324 & $3^{\text {a Relación }}$ & Chalcas vs. Tlacochcalcas \\
& $5^{\text {a }}$ Relación & \\
& $7^{\text {a }}$ Relación & \\
1368 & $3^{\text {a }}$ Relación & Chalcas vs. Mexicas \\
1375 & $7^{\text {a Relación }}$ & \\
1380 (ca.) & & \\
1381 & $6^{\text {a Relación }}$ & Chalcas vs. Tepanecas
\end{tabular}


motivo, nos informa tanto de los acontecimientos históricos de su pueblo de origen, como de algunos sucesos del reino mexica. $\mathrm{La}$ obra, de hecho, termina con el relato de la Conquista y del primer período colonial.

En la fuente chalca se mencionan algunos enfrentamientos llamados xochiyaoyotl precedentes al auge del imperio azteca. En la tercera Relación se menciona una guerra florida en que estaban implicados los chalcas y los tlacochcalcas en el año 1 tecpatl (1324):

1 tecpatl xihuitl, 1324. Nican ypan in motlahtocatlalli yn huehue Acamapichtli, yn Colhuacan tlahtohuani. Auh no yquac yn ipan in xihuitl yquac peuhqui yn xochiyaoyotl yn ompa Chalco; yehuantin quichiuhque yn chalca yhuan tlacochcalca (Castillo 1991:61).

Año 1 técpatl, 1324. Aquí en éste se asentó en el mando el huehue Acamapichtli, tlahtohuani de Colhuacan. Y también durante este año fue cuando tuvo comienzo la xochiyaoyotl allá por Chalco; la hicieron los chalca y tlacochcalca (Castillo 1991:67).

En la quinta Relación, Chimalpahin proporciona más detalles acerca de este acontecimiento:

I Tecpatl xihuitl, 1324. Ypan in yn peuhqui xochiyaoyotl, auh yn ihquacmacatlalliaya yn tlacochcalca yn i mochintin yn chalca yn ixpan in diblo. Yn omacatlallique niman ye momamimictia, yuhqui yn nahuatil yuhqui ynnepohual, nocuel onmocahua, çan iuhqui ynic mahuiltiaya. Auh çatepan in yequene quimococolchihuillia; çanno yn neacatlalliayan mopehualtique in ye momamimictia, yequene ye quimococolchihuillia, ye quauhtlamatzayanaltica mohuitequi ye quauhtlatzayantica momotla; cenca ye quincocohua yn tlacochcalca, miequintin in ye temac miqui. Niman ye hualmoquetza yn teomama yn itoca Quetzalcanauhtli, ye tlahtohua ye quimilhuia yn chalca: "Tle amay, chalcayé? Huelle ye anmonelchihua; cuix amo çan tonahuatil çan tonepohual yn toneacatlalliaya titomamimictizque? Auh $y-\| l[137 v]-n$ axcan in ye anmonelchihuaznequi, auh yn at ye yuh yn at ye anmonelchihua; oc huel ximononotzacan!". Yc hualtetlacahualti yn Quetzalcanauhtli. Xixinque, yahyahque; ye yc cenpeuhtica yn yn cocolotl yn xochiyaoyotl. Auh yn tlacochcalca yn onmatlacuilliaya yn incihuahuan, ymicanpa yn quinmochilliaya yn imatlacuizcon; auh yn omoquaquahuilliaya yn inpilhuan, ymicampa yn quimontleminilliaya yn inquauhtzin. Cenca yequene quinquequellohua yn tlacochcalca. Auh ynic quintocaque yn mexica in yancuican yn culhuaque, oncan motlallito yn Nexticpac, cexiuhtique yn oncan yn ipan in $\mathrm{Ce}$ Tecpatl xihuitl, yehuatl quinyacantihuitz yn Tenochtzin. Yn Ce Tecpatl xihuitl yn no yehuatl ypan motlahtocatlalli in tlacatl Huehue Acamapichtli tlahtohuani Culhuacan; ynin ypiltzin yn Coxcoxtli. Quizqui yn Ce Tecpatl xihuitl (Tena 1998:358-360).

1 Técpatl, 1324. En este año se iniciaron las guerras floridas, al tiempo en que los tlacochcalcas y todos los chalcas solían sentarse sobre cañas delante del Diablo. Después de sentarse sobre las cañas se golpeaban con las manos, según su costumbre y tradición, pero luego se detenían, pues lo hacían sólo como por diversión. Pero a la postre acabaron haciéndose daño; después de haberse sentado sobre las cañas comenzaron a golpearse con las manos, pero terminaron haciéndose daño, porque se golpearon y se hirieron [también] con ramas de árbol; maltrataron mucho a los tlacochcalcas, y muchos [de ellos] murieron a manos de los contrarios. Enseguida se levantó el teomama Quetzalcanauhtli, y hablando a los chalcas les dijo: "¿Qué hacéis, oh chalcas? Estáis introduciendo novedades; pues ¿acaso no es nuestra costumbre y tradición el sentarnos sobre cañas y luego [sólo] golpearnos con las manos? Pero ahora vosotros pretendéis introducir novedades, o quizá ya las habéis introducido; ;reflexionad sobre esto!”. Así trató de disuadirlos Quetzalcanauhtli. Se dividieron, se enemistaron; así comenzó este mal de las guerras floridas. Y cuando las mujeres de los tlacochcalcas iban a traer agua, por detrás les apedreaban los cántaros; y cuando sus hijos iban a traer leña, por detrás les quemaban la leña. En fin, se hacía gran burla de los tlacochcalcas. Cuando los colhuas persiguieron nuevamente a los mexicas, éstos se fueron a asentar en Nextícpac, donde se quedaron durante este año de 1 Tecpatl, mientras los venía conduciendo Tenochtzin (Tena 1998:359-361).

Según se destaca en el testimonio de Chimaplahin, en 1324 empieza un enfrentamiento entre los chalca-acxotecas y los tlacochcalcas. Los tlacochcalcas, después de su llegada en el territorio de Tlalmanalco, aumentan mucho su red de alianzas con los pueblos chalcas ya presentes en él, pero también causándose enemistad con otros pueblos, en particular con los acxotecas (Navarrete Linares 2000). En este primer caso se le denomina xochiyaoyotl a un enfrentamiento ritual. Los chalcas y los tlacochcalcas, en aquel tiempo, estaban involucrados en una ceremonia ritual, lo que nos hace pensar en una guerra simulada (Hicks 1979). Notamos que la xochiyaoyotl se describe como un juego con una finalidad ritual, pero que no incluye sacrificios humanos. Sin embargo, el conflicto ritual se convierte en algo más serio en que los chalcas se aprovecharon de la ocasión para herir y matar a los tlacochcalcas. El teomama Quetzalcanauhtli, portador del dios tlacochcalca Tetzcatlipoca, intenta poner término a esa violencia, pero sin éxito. Durante casi ocho años los tlacochcalcas siguen padeciendo vejaciones, hasta que en 1332 Tetzcatlipoca ordena a Quetzalcanauhtli la salida de los tlacochcalcas, los cuales parten rumbo a Coyoacán (Navarrete Linares 2000).

También en la séptima Relación, se menciona otra guerra florida entre tepanecas y chalcas, en el año 1381. Sin embargo, Chimalpahin no proporciona mayores detalles:

VI calli xihuitl, 1381 años Ypan ynin compehualtique in Tepaneca yhuan yn Chalca yn xochiyaoyotl, yn iuh quihtohua yehuantin Tlacochcalca Chalca yn itech ynxiuhtlapohual (Siméon 1889:72).

Año 6 calli, 1381 años En éste hicieron comenzar los tepaneca y los chalcas la xochiyaóyotl; así lo dicen ellos, los tlacochcalca chalca, en su cuenta de años (García Quintana 2003:67).

Anné 6 maison, 1381. Alors les Tépanàeques et les Chalcas entreprirent la guerre des fleurs, ainsi que l'indiquent les Tlacochcalcas Chalcas dans leurs annales (Siméon 1889:73).

Por el contrario, las descripciones de los enfrentamientos entre chalcas y mexicas nos ofrecen algunos detalles interesantes. En el año 1376 de la tercera Relación podemos leer:

i tecpatl xihuitl, 1376 Ypan in, yn cequintin huehuetque yn iuh quimachiyotia, yn motlahtocatlalli Acamapichtli yn Tenochtitlan. Auh no yquac in ipan in xihuitl in acito yaoyotl yn ompa Chalco Atenco; auh chicuexihuitl yn çan oc xochiyaoyotl yn manca, yn quimacia yn mexicapipiltin, çan oc quincahuaya yn chalca; auh no yhui, yn quimacia yn chalca auh no yhui, yn quimacia yn chalcapipiltin çan oc quincahuaya yn mexica; ca çan ic ynceltin yn macehualtin yn miquia (Castillo 1991:102).

Año 1 tecpatl, 1376 En éste, tal como señalan algunos antiguos, fue en el que Acamapichtli se asentó en el mando en 
Tenochtitlan. Y también durante este año fue a llegar la guerra allá por Chalco Atenco; pero en los ocho años que aún permaneció la xochiyaóyotl, a los pipiltin mexica que alcanzaban todavía los dejaban los chalcas, y de la misma manera, a los pipiltin chalca que alcanzaban todavía los dejaban los mexica; por entonces únicamente los macehuales morían (Castillo 1991:103).

Lo mismo atestigua la séptima Relación al mismo año:

1 tecpatl xihuitl, 1376 años. Ypan inyn acito xochiyaoyotl yn ompa Chalco Atenco, yn iuh quitoa Amaquemeque, chicuexihuitl ycan oc xochiyaoyotl yn manca, yn quimacia yn Mexica pipiltin, çan oc quincahuaya yn Chalca, mocuepaya yn inchan Mexico. Auh no yhui, yn quimacia yn Chalca pipiltin çan oc quincahuaya yn Mexica, mocuepaya yn inchan Chalco. Ca çan oc yn celtin yn macehualtin yn miquia (García Quintana 2003: 64).

Año 1 tecpatl, 1376 años En éste llegó la xochiyaóyotl allá en Chalco Atenco así lo dicen los amaquemeque, que ocho años, hasta ahora, permanecía la xochiyaóyotl. [Cuando] alcanzaban a los pipiltin mexica, los chalca aún los dejaban, se regresaban a su lugar en Mexico. Y también de la misma manera [cuando] alcanzaban a los pipiltin chalca, los mexica aún los dejaban, se regresaban a su lugar en Chalco; únicamente ellos solos, los macehuales, morían (García Quintana 2003:65).

Dejando por el momento al lado las implicaciones de carácter cronológico, analizamos las informaciones de tipo tácticoorganizativo que Chimalpahin nos proporciona. Tanto en la tercera como en la séptima Relación, se menciona que únicamente los macehualtin (los del pueblo) morían, mientras que los pipiltin (los nobles) eran liberados al momento en que eran capturados. La séptima Relación precisa que los pipiltin chalcas y mexicas eran también liberados tras su captura, y que todos regresaban a sus respectivos pueblos.

Estos pasajes parecen describir una guerra hecha de mutuo acuerdo cuya finalidad no era ni la de obtener cautivos ni, tampoco, la de matar. Incluso la mención de la muerte de los macehualtin parece tener un matiz fortuito. Ningún tipo de sacrificio se menciona, lo que sugiere enfrentamientos orientados simplemente a la práctica militar (Hicks 1979). Según asevera Hicks, en los pasajes de la obra de Chimalpahin donde se describen los sacrificios humanos, los cautivos proceden siempre de la guerra de conquista, la cocoltic yaoyotl, y nunca de la xochiyaoyotl. El planteamiento de Chimalpahin no contrasta con la ideología nahua ni con la de las demás culturas. Considerando el papel sagrado de la guerra y los sacrificios humanos en las sociedades mesoamericanas, parece raro que los cautivos a sacrificar se obtuvieran por medio de simples guerras pactadas. Es más verosímil que, puesta la sacralidad del oficio de la guerra y de la conquista, los cautivos destinados al sacrificio procedieran más bien de la cocoltic yaoyotl.

La misma atestación acerca del enfrentamiento entre chalcas y mexicas del año 1376 se encuentra en los Anales de Cuauhtitlan:

1 tecpatl. En este año empezaron los mexicanos y chalcas a escaramuzar unos con otros; aún no se trababan ni se mataban; sino que todavía parecía que jugaban. Eso es lo que se llama guerra florida. Nueve años duró en Techichco de Colhuacan el juego de los mexicanos y chalcas (Velázquez 1975b:32).

Es interesante notar que los Anales de Cuauhtitlan (Velázquez 1975b) utilizan la palabra "juego" para indicar las guerras floridas, así como Chimalpahin utiliza la palabra “diversión”, a propósito del año 1324. Este testimonio nos muestra que los mexicas estaban involucrados en los enfrentamientos llamados xochiyaoyotl antes de que empezara el reino de Motecuhzoma Ilhuicamina, bajo cuyo reinado se fija la invención de la práctica de la guerra florida. Por lo menos, cabe admitir que los mexicas, los chalcas y probablemente todos los demás pueblos mesoamericanos, ya conocían el concepto de xochiyaoyotl.

Pasamos ahora al análisis de las implicaciones de carácter histórico y cronológico que las descripciones de Chimalpahin nos plantean. Las Relaciones presentan fechas diferentes y muchas veces contradictorias de los enfrentamientos entre chalcas y mexicas respecto tanto a la xochiyaoyotl como a la cocoltic yaoyotl (ver Tabla 2). Un estudio puntual de estas contradicciones, junto con un tentativo de solución podrá ayudar a formular una interpretación más exhaustiva del tema. Tanto la tercera como la séptima Relación revelan que en el año 1 acatl (1415) termina la xochiyaoyotl y empieza la cocoltic yaoyotl entre chalcas y mexicas, puesto que ambas partes se empiezan a matar también a los pipiltin. Sin embargo, mientras que la tercera Relación nos indica que la xochiyaoyotl duró cuarenta años (dando el año 1375 como comienzo de la guerra florida), la séptima Relación nos ofrece dos fechas:

\section{Año 1 acatl, 1415 años}

$\mathrm{Y}$ ya en este año vino a moverse la guerra en Atenco. Finalmente se asentó del tondo el enojo; produco cólera la guerra. Allí desapareció la xochiyaóyotl. Al final ya no dejaban los pipiltin mexica a los chalca que cogían, y también así los pipiltin chalca, a los mexica que cogían ya no los dejaban. Allí se perdió la que se dice xochiyaóyotl que había permanecido cuarenta años. Y aunque así lo dicen los tlacochcalca chalca, la xochiyaóyotl había permanecido sólo treinta y cinco años (García Quintana 2003:46).

I acatl xihuitl, 1415 años. Auh ye ipan inyn huallolin yaoyotl yn Atenco in yequene cen motlali cuallantli yn cocoltic yaoyotl; oncan poliuh in xochiyaoyotl, yn aocmo yequene quincahuaya in Mexica pipiltin yn quimacia Chalca, auh no yhui yehuantin yn Chalca pipiltin yn quimacia yn Mexica aocmo quincahuaya; oncan in poliuh in motenehua xochiyaoyotl yn manca ompohualxihuitl. Auh macihuiyn iuh yehuantin quihtohua Tlacochcalca Chalca yn manca xochiyaoyotl çan cempohualloncaxtolli xihuitl, yn iuh ypan in quimachiyotia in ynueue xiuhtlapohualamauh (García Quintana 2003:47)

Según la fuente tlacochcalca, la xochiyaoyotl dura treinta y cinco años, con lo que remonta a 1380 como fecha de inicio de la guerra florida. La fuente chalca, por su parte, revela que la misma guerra duraba desde hace cuarenta años; es decir, un inicio en 1375. Sin embargo, tanto en la sexta como en la séptima Relación se señala el año 1376 como fecha de inicio de la guerra florida en Chalco. Según subraya Durand-Forest (1987), la contradicción se explica por el hecho de que en el año 1376, tanto la tercera como la séptima Relación indican que la xochiyaoyotl duraba desde hace ocho años; este cálculo remonta su inicio al año 1368. De hecho, la séptima Relación en el año 1367 nos informa de una guerra entre los itzapallapanecas y tepanecas.

Es razonable considerar que la fecha de 1376 que nos propone Chimalpahin sea la fecha del inicio, en Chalco, de una xochiyaoyotl que ya estaba en curso en el Altiplano. Para apoyar esta afirmación, cabe destacar que en el mismo año 1376 se menciona el comienzo del mando de Acamapichtli como primer rey de MexicoTenochtitlan, lo que nos hace pensar que los mexicas antes no se 
Tabla 2. Eje cronológico de los cómputos de las guerras floridas entre chalcas y mexicas, según las Relaciones de Chimalpahin

\begin{tabular}{llll}
\hline \hline 1324 & $1368 / 1367$ & 1376 & $1380 / 1381$ \\
\hline $\begin{array}{l}\text { Xochiyaoyotl entre } \\
\text { chalcas y tlacochcalcas }\end{array}$ & $\begin{array}{l}\text { Xochiyaoyotl entre } \\
\text { chalcas y mexicas }\end{array}$ & $\begin{array}{l}\text { Xochiyaoyotl entre chalcas y } \\
\text { mexicas según la fuente chalca }\end{array}$ & $\begin{array}{l}\text { Xochiyaoyotl entre chalcas y } \\
\text { mexicas según la fuente } \\
\text { tlacolchcalca }\end{array}$ \\
\hline \hline
\end{tabular}

incorporaban a la xochiyaoyotl, por ser recién llegados en el territorio. Según destaca Durand-Forest (1987), probablemente los diferentes cómputos acerca de las guerras floridas indican en realidad un continuum de enfrentamientos en los que de vez en vez, tomaban parte los diferentes pueblos. Esta interpretación podría resolver la diferencia en el cálculo de las fechas por parte de los atltepetl, los cuales probablemente contaban los años a partir de su propia incorporación a la lucha. La misma interpretación está compartida por García Quintana (2003) que, al referirse al año 1381 ya citado, comenta que si los tlacochcalcas indican este año como comienzo de la xochiyaoyotl entre tepanecas y chalcas es porque probablemente consideraron el año en que ellos se incorporaron a una lucha en la cual otros chalcas ya estaban actuando desde antes.

El modo en que Chimalpahin representa a la xochiyaoyotl entre chalcas y mexicas sugiere enfrentamientos pactados en los que el fin no era matar ni capturar víctimas para sacrificios. Esas guerras se describen como si fueran una práctica militar cuya finalidad probable era también la diversión, dado que los cautivos siempre se liberaban. Cuando los cautivos no eran liberados o algunos de los pipiltin eran muertos, Chimalpahin ya no habla de xochiyaoyotl, sino de cocoltic yaoyotl. La práctica militar es, como indicamos páginas arriba, una de las motivaciones ofrecidas por Tlacaelel y Motecuhzoma Ilhuicamina al pactar la feria militar contra los tlaxcaltecas. Curiosamente, este aspecto del ejercicio militar no se ha considerado mucho en los estudios acerca de la xochiyaoyotl, los cuales han privilegiado más bien el rasgo religioso-ritual (Hicks 1979).

Según el análisis proporcionado en este estudio, es probable que los mexicas entran en contacto con la práctica de la xochiyaoyotl asociándola al puro ejercicio militar, de gran importancia en su propia cultura. Basta con pensar en el énfasis que Bernardino de Sahagún (1540) proporciona a dicho ejercicio a la hora de describir la educación de los niños mexicas, tanto de la nobleza como del pueblo. Es posible que la ideología mexica se haya apropiado de la práctica de la guerra florida y adaptado su uso a las nuevas necesidades políticas después de que el reino azteca hubiere alcanzado su auge en el Valle de México. Gracias a esta lectura de los hechos, podemos aceptar también las verosímiles interpretaciones de Bueno Bravo (2009) y de Hassig (1992), los cuales destacan también una función propagandística de la xochiyaoyotl. Según los dos, las guerras floridas mostraban también cierta superioridad a potenciales enemigos y les daba una idea del posible escenario si se instituyera una guerra real.

Hay que subrayar que los mexicas muchas veces se habían ya apropiado de y construido sobre algunos aspectos de la ideología zuyuana. Según Domenici (2005), hay ejemplos del modo en que la propaganda política azteca reelaboraba antiguos conceptos mesoamericanos bajo una nueva doctrina. Según Domenici (2005), el escenario de la conquista imperial en la Piedra Tizoc, el cuauhxicalli descubierto durante las excavaciones en Tenochtitlan en 1970, seria uno de estos ejemplos. El bajorrelieve presenta al soberano que tiene por los cabellos un cautivo, el cual lleva junto el topónimo de la ciudad conquistada. Este motivo es conocido; sin embargo, Domenici subraya que la novedad está constituida en el carácter divino de los personajes.

El ganador lleva los emblemas de Tetzcatlipoca (el pie humeante) y de Huitzilopochtli (la gorra con el colibrí), mientras que el derrotado lleva los del dios protector de la ciudad conquistada. La guerra de conquista se convierte, por lo tanto, en un choque entre divinidades y se coloca en un ámbito evidentemente sobrenatural (Domenici 2005:189). Los aztecas se consideran el pueblo electo para sujetar el mundo al orden de que se sienten portadores. Esta ideología, a través de una reelaboración y superación de las viejas concepciones zuyuanas, se convierte en un fundamental instrumento de legitimación de su proyecto expansionista (Domenici 2005:190). A la luz de las últimas consideraciones, por lo tanto, no es razonable rechazar por completo las recientes teorías revisionistas de Isaac (1983), Hassig (1992), y Bueno Bravo (2009), las cuales describen el uso de la xochiyaoyotl como legitimación religiosa al propósito expansionista del imperio azteca.

\section{CONCLUSIONES}

La sacralidad del oficio de la guerra no fue prerrogativa exclusiva de los aztecas, sino una constante de todos los grandes estados mesoamericanos. Ya en Teotihuacan, el primer grande imperio de Mesoamérica la ciudad más grande del Valle de México en 100 d.C., se encuentran atestaciones de una guerra de tipo religioso-ritual. Teotihuacan se jactaba de una formidable armada, aunque eso no significó el intento de llevar adelante un plan de conquista universal similar al de los mexicas. A pesar de la tradicional visión que considera el periodo Clásico como un período de paz, en Teotihuacán existen evidencias del enterramiento de guerreros sacrificados bajo el Templo de Quetzalcóatl que se remontan a 150 d.C. (Hassig 1992:60).

Por lo que concierne a la guerra florida, son muchas las atestaciones acerca de esta práctica en la cultura mexica, mientras que resultan muy escasas aquellas relativas a tiempos precedentes. Además, faltan muchos datos sobre la percepción de la xochiyaoyotl por parte de los demás pueblos del Valle tlaxcalteco-poblano. Sin embargo, a la luz del descubrimiento de las nuevas fuentes mencionadas a lo largo de este trabajo y gracias a los nuevos estudios al respecto, es indispensable suponer que la xochiyaoyotl ha sido muchas cosas de acuerdo al contexto temporal preciso, en que se desarrolló. La consideración de esta perspectiva histórico-temporal precisa es indispensable.

Sin embargo, se puede rechazar por completo una interpretación ritual de la guerra florida en la cultura mexica, sobre todo durante el reino de Motecuhzoma II. Esto no implica que esta interpretación pueda ser extendida al punto de vista tlaxcalteco que, según las fuentes, no comparte la ideología nahua. Asimismo, no es de rechazar por completo la consideración de una xochiyaoyotl como simple ejercicio militar, como sugiere la 
obra de Chimalpahin. Después de la caída de Teotihuacán, la tradición zuyuana llega al altiplano maya y a la cultura zapoteca, alcanzando su auge en la ideología mítico-religiosa del imperio azteca. El discurso de Tlacaelel acerca de la institución de la feria militar contra los estados del Valle tlaxcalteco-poblano, plantea una ideología para evitar la decadencia tolteca que habría impresionado a los pueblos mesoamericanos. La guerra, en la ideología mexica, representaba uno de los medios para preservar el orden universal (Zantwijck 1962).

Además, si contamos con el testimonio de Chimalpahin, la guerra florida parece nacer propiamente en la cultura chalca. Sólo en la segunda mitad del siglo XIV los mexicas, recién llegados en el Valle de México, se incorporan a la cultura que allí encuentran y se involucran también en las llamadas xochiyaoyotl. A la hora de alcanzar su fulgor, es razonable pensar que el reino mexica se haya apropiado del oficio de la xochiyaoyotl, sujetándolo a su propio intento imperialista que necesitaba, evidentemente, de una constante justificación tanto a nivel religioso como a nivel jurídico.

En conclusión, cabe aceptar que no es tarea fácil estudiar e interpretar de modo exhaustivo un tema así complejo como la xochiyaoyotl. Aún faltan elementos, evidencias históricas y sobre todo más fuentes que permitan una comparación completa de los diferentes puntos de vista. Con este trabajo esperamos haber contribuido por lo menos a plantear nuevas preguntas y nuevas ocasiones de investigación acerca de un tema tan fascinante y todavía tan controvertido.

\section{RESUMEN}

The flowery wars have always been considered as a long-lasting conflict between the Aztec Triple Alliance and the reigns of the Valley of Puebla and Tlaxcala. For years, the xochiyaoyotl has been studied as a ritual war extended within time and based on an agreement between the two parts involved. All the theories and primary sources agree with the ritual interpretation, claiming that the purpose of the xochiyaoyotl was to obtain victims to sacrifice to the Gods. However, from the last decades of the twentieth century, thanks to the discovery and translation of new documents, the mere ritual and religious concept of the flowery war has been called into question. The source that gives us the most interesting details about this conflict is the work by the Chalca historian Domingo Francisco de San Antón
Muñón Chimalpahin Quauhtlehuanitzin. Chimalpahin's testimony discusses wars called xochiyaoyotl that occurred before the reign of Motecuhzoma Ilhuicamina. These conflicts involved the Chalcas and Tlacochcalcas before the Mexicas. Furthermore, Chimalpahin never mentions sacrifices from the flowery war. When he talks about sacrifices he always refers to the cocoltic war, which is, according to him, the conquest war. By means of a deep analysis of Chimalpahin's testimony, this paper aims to question the theory of the invention of the flowery war during the reign of Motecuhzoma Ilhuicamina and to consider the hypothesis by which the Aztecs made themselves masters of the xochiyaoyotl practice, developing it according to the new political needs of the empire.

\section{REFERENCES}

Alvarado Tezozómoc, Hernando

1975 Crónica Mexicana. Editado por Manuel Orozco y Berra. Editorial Porrúa, México.

1987 Crónica Mexicana, precedida del Códice Ramírez manuscrito del siglo xvi intitulado: Relación del origen de los indios que habitan esta Nueva España según sus historias. $4^{\mathrm{a}}$ edición, anotada con estudio cronológico por Manuel Orozco y Berra. Editorial Porrúa, México.

Benoist, Valérie

2003 La construcción de una comunidad nahua/española en las Relaciones de Chimalpahin. Estudios de Cultura Náhuatl 34:205-218.

Bueno Bravo, Isabel

2009 Las guerras floridas. Revista de Historia Militar 106:11-34.

Caso, Alfonso

1927 El teocalli de la Guerra Sagrada. Talleres Gráficos de la Nación, México.

1961 Il popolo del sole. Il Saggiatore, Milano.

Caso, Alfonso e Ignacio Bernal

1952 Urnas de Oaxaca, Vol. 2. Memorias del Instituto Nacional de Antropología e Historia, México.

Chimalpahin Quauhtlehuanitzin, Domingo

1889 Annales. Traducido y editado por Rémi Siméon, 13 vols. Maissonnueve et Ch. Leclerc Éditeurs, Paris.

1987 Trosième relation de Chimapahin Quauhtlehuanitzin. Traducido y editado por Jacquelin de Durand-Forest, Paris, L'Harmattan.

1991 Memorial breve acerca de la fundación de la ciudad de Culhuacan. Traducido y editado por Víctor Castillo. Universidad Autónoma de México, México.

1997 Primer Amoxtli. $3^{a}$ Relación de las différentes histoires originales. Traducido y editado por Victor Castillo. Universidad Autónoma de México, México.

1998 Las ocho relaciones y el memorial de Culhuacán. Traducido y editado por Rafael Tena. 2 vols. Consejo Nacional para la Cultura y las Artes, Cien de México, México.

2003 Séptima Relación de las Différentes Histoires Originales.

Traducido y editado por Josefina García Quintana. Universidad Autónoma de México, México.

Davies, Nigel

1966 Los señoríos independientes del imperio azteca. Instituto Nacional de Antropología e Historia, México.

Díaz del Castillo, Bernal

1968 Historia verdadera de la conquista de la nueva España. Espasa-Calpe, Madrid.

Domenici, Davide

2005 I linguaggi del potere. Jaca Book, Milano.

Durán, Diego

1967 Historia de la Indias de Nueva España e islas de Tierra Firme. Edición de Ángel María Garibay. 2 vol. Editorial Porrúa, México.

1980 Libros de los ritos y fiestas de los antiguos mexicanos. Editado por César Mecazaga Ordono. Editorial Innovación, México.

Durand-Forest, Jacqueline de

1987 L'Histoire de la vallée de Mexico selon Chimalpahin Quauhtlehuanitzin, Vol. 1. L'Harmattan, Paris.

Garibay, Ángel María

1965 Historia de los Mexicanos por sus pinturas. En Teogonía e historia de los mexicanos. Tres opúsculos del siglo XVI, editado por Ángel María Garibay, pp. 23-66. Editorial Porrúa, México.

González González, Carlos Javier

2011 Xipe Tótec. Guerra y regeneración del maíz en la religión mexica. Fondo de Cultura Económica, México.

Harner, Michael

1977 The Ecological Basis for Aztec Sacrifice. American Ethnologist 4: $117-135$

Hassig, Ross

1981 The Famine of 1 Rabbit: Ecological Causes and Social Consequences of a Pre-Columbian Calamity. Journal of Anthropological Research 37: $172-182$.

1992 War and Society in Ancient Mesoamerica. University of California Press, Berkeley. 
Hicks, Frederic

1979 "Flowery War" in Aztec History. American Ethnologist 6:87-92. Isaac, Barry

1983 The Aztec "Flowery War": A Geopolitical Explanation. Journal of Anthropological Research 39:415-432.

Ixtlilxochitl, Fernando de Alva

1985 Historia de la nación chichimeca. Editado por Germán Vázquez. Serie Crónica de América, Vol. II. Historia 16, Madrid.

Messiaen, Sam

2003 Some Interesting Observations on Chimalpahin by Use of His "Diferentes historias originales." Estudios de Cultura Náhuatl 34:218-256.

Muñoz Camargo, Diego

1892 Historia de Tlaxcala. Tipografía de la Secretaria de Fomento, México.

Navarrete Linares, Federico

2000 Mito, historia y legitimidad política: las migraciones de los pueblos del Valle de México. Tesis doctoral, Facultad de Filosofía y Letras, Universidad Autónoma de México, México.

Pomar, Juan Bautista

1975 Relación de Tetzcoco. Editorial Libros de México, México.

Romero Galván, José Rubén

1976 Posible esquema de las Diferentes historias originales de Chimalpahin. Estudios de Cultura Náhuatl 12:73-78.

1977 Las fuentes de las Diferentes historias originales del Chimalpahin. Journal de la Société des Américanistes 64:51-56.

Sahagún, Bernardino de

1989 Códice Florentino: Historia general de las cosas de nueva España: primera versión íntegra del texto castellano del manuscrito conocido como Códice Florentino. Editado por Alfredo López Austin y Josefina García Quintana. Alianza Editorial Mexicana, México.

Soustelle, Jacques

1965 Vita quotidiana degli aztechi. Il Saggiatore, Milano.

Tapia, Andrés de

1858 Relación de algunas cosas de las que acaecieron al muy ilustre señor don Hernando cortés, Marques del Valle, desde que se determinó ir a descubrir tierra en la tierra firme del mar Océano. En Documentos para la historia de México, Vol. 2, editado por Joaquín García Izcalbalceta, pp. 128-594. Kraus Reprint, Nendeln (Liechtenstein).

Taube, Karl

1992 The Temple of Quetzalcoatl and the Cult of Sacred War at Teotihuacan. RES: Anthropology and Aesthetics 21:53-87.

Velasco Murillo, Dana

2016 "For the Last Time, Once and for All": Indians, Violence, and Local Authority in the Colonial City, Zacatecas, Mexico, 1587-1628. Ethnohistory 63:47-70.

Velázquez, Primo F. (trans. and ed.)

1975a Leyenda de los soles. En Códice Chimalpopoca. Anales de Cuauhtitlán y Leyenda de los soles, traducido y editado por Primo F. Velázquez, pp. 119-162. Universidad Autónoma de México, México.

1975b Anales de Cuauhtitlan. En Códice Chimalpopoca, editado y traducido por Primo F. Velásquez, pp. 1-118. Universidad Autónoma de México, México.

Zantwijk, Rodolfo van

1962 La paz azteca. Ordenación del Mundo por los Mexica. Estudios de Cultura Náhuatl 3:101-137. 